\title{
TGF $\beta 1$ single-nucleotide polymorphism C-509T alters mucosal cell function in pediatric eosinophilic esophagitis
}

\author{
L. D. Duong ${ }^{1,2}$, R. Rawson ${ }^{1,2}$, A. Bezryadina ${ }^{3}$, M. C. Manresa ${ }^{1,2}$, R. O. Newbury ${ }^{4}$, R. Dohil ${ }^{2,5}$, Z. Liu $^{3}$, K. Barrett ${ }^{5,6}$, R. Kurten ${ }^{7}$ and $^{2}$ \\ S. S. Aceves $1,2,6$
}

Eosinophilic esophagitis (EoE) is a chronic Th2 antigen-driven disorder associated with tissue remodeling. Inflammation and remodeling lead to esophageal rigidity, strictures, and dysphagia. TGF $\beta 1$ drives esophageal remodeling including epithelial barrier dysfunction and subepithelial fibrosis. A functional SNP in the TGF $\beta 1$ gene that increases its transcription (C-509T) is associated with elevated numbers of esophageal TGF $\beta 1$-expressing cells. We utilized esophageal biopsies and fibroblasts from TT-genotype EoE children to understand if TGF $\beta 1$ influenced fibroblast and epithelial cell function in vivo. Genotype TT EoE esophageal fibroblasts had higher baseline TGF $\beta 1$, collagen 1a1, periostin, and MMP2 $(p<0.05)$ gene expression and distinct contractile properties compared with CC genotype ( $n=6$ subjects per genotype). In vitro TGF $\beta 1$ exposure caused greater induction of target gene expression in genotype CC fibroblasts $(p<0.05)$. Esophageal biopsies from $\Pi$-genotype subjects had significantly less epithelial membrane-bound E-cadherin $(p<0.01)$ and wider cluster distribution at nanometer resolution. TGF $\beta 1$ treatment of stratified primary human esophageal epithelial cells and spheroids disrupted transepithelial resistance $(p<0.001)$ and E-cadherin localization $(p<0.0001)$. A TGF $\beta 1$-receptor-I inhibitor improved TGF $\beta 1$-mediated E-cadherin mislocalization. These data suggest that EoE severity can depend on genotypic differences that increase in vivo exposure to TGF $\beta 1$. TGF $\beta 1$ inhibition may be a useful therapy in subsets of EoE patients.

Mucosal Immunology (2020) 13:110-117; https://doi.org/10.1038/s41385-019-0214-9

\section{INTRODUCTION}

Eosinophilic esophagitis (EoE) is an antigen-driven chronic Th2 disease of increasing prevalence. ${ }^{1-3}$ Chronic inflammation causes esophageal remodeling with end-organ rigidity, narrowing, and dysmotility with clinical dysphagia and food impaction. ${ }^{4-8}$ Despite the success of elimination diet and topical esophageal corticosteroid therapies, some patients remain unresponsive or break through standard medical interventions, and progress to disease complications. ${ }^{9-12}$ The disease mechanisms that underlie therapy nonresponsive and/or severe EoE are not clearly understood.

Previous studies have demonstrated that there are genetic predispositions to EoE onset. These include specific genotypes or mutations in thymic stromal lymphopoeitin and its ${ }^{13}$ receptor, ${ }^{14,15}$ calpain $14,{ }^{16}$ SPINK5/7, ${ }^{17}$ and TGF $\beta 1 .{ }^{18,19}$ In addition, genotypic variations can associate with patient phenotypes in EoE. The EoE TSLP risk allele can alter patient phenotype and associate with increased EoE food triggers. ${ }^{13}$ Children who have TGF $\beta 1$ genotype $\Pi$ at the functional promoter single-nucleotide polymorphism (SNP) C-509T have increased numbers of TGF $\beta 1$-expressing cells in the lamina propria and more severe histologic disease, including fibrosis, especially in the context of food sensitization. ${ }^{20,21}$ In asthma, genotype $\Pi$ at -509 can associate with asthma severity in hildren living close to freeways, suggesting a gene-environment interaction. $^{22,23}$

Mechanistic studies demonstrate that $\Pi$ genotype at C-509T increases TGF $\beta 1$ gene transcription either through the loss of negative regulation via AP-1 or through positive regulation by YY$1{ }^{24}$ TGF $\beta 1$-positive cells are elevated in EoE subjects, especially in genotype $\Pi$, and genetic syndromes that alter the balance of TGF $\beta 1$ and its signals in vivo, such as Loeys-Dietz and Marfan's, are associated with EoE. ${ }^{18-21}$ These findings suggest that the in vivo balance and timing of TGF $\beta 1$ expression is likely critical for proper esophageal function. Indeed, TGF $\beta 1$ has a myriad of effects in the EoE esophagus. It causes esophageal epithelial-tomesenchymal transition, myofibroblast transformation, myocyte and fibroblast contraction, loss of claudin-7 with epithelial barrier dysfunction, and fibrotic gene expression. ${ }^{25-29}$ Given these effects, it is clinically impactful to understand if EoE patients who have endogenously elevated TGF $\beta 1$ have altered structural cell function. In this scenario, there could be an opportunity for personalized medicine via TGF $\beta 1$-altering therapies in subsets of patients. Herein, we utilized esophageal fibroblasts and biopsies from patients of $T \mathrm{~T}$ and $C \mathrm{C}$ genotypes at C-509T to understand the potential detrimental effects of increased esophageal TGF $\beta 1$ exposure in vivo.

${ }^{1}$ Division of Allergy \& Immunology, University of California, San Diego, San Diego, CA, USA; ${ }^{2}$ Department of Pediatrics, University of California, San Diego and Rady Children's Hospital San Diego, San Diego, CA, USA; ${ }^{3}$ Department of Bioengineering, University of California, San Diego, San Diego, CA, USA; ${ }^{4}$ Department of Pathology, University of California, San Diego, Rady Children's Hospital, San Diego, CA, USA; ${ }^{5}$ Division of Gastroenterology, San Diego, CA, USA; ${ }^{6}$ Department of Medicine, University of California, San Diego, San Diego, CA, USA and ${ }^{7}$ Arkansas Children's Research Institute and University of Arkansas for Medical Sciences, Little Rock, AK, USA Correspondence: S. S. Aceves (saceves@ucsd.edu)

Received: 12 March 2019 Revised: 18 September 2019 Accepted: 24 September 2019

Published online: 21 October 2019 
TGF $\beta 1$ single-nucleotide polymorphism C-509T alters mucosal cell function...

L.D. Dong et al.

111

RESULTS

Genotype $\Pi$ and CC fibroblasts are phenotypically different We utilized esophageal fibroblasts from 12 pediatric EoE subjects (6 genotypes of TT, 6 genotypes of CC) (see Table 1 for clinical characteristics). We assessed baseline TGF $\beta 1$ gene expression in passage and disease activity-matched fibroblasts from genotype CC and TT patients. All subjects had active EoE as defined histologically at the time of fibroblast isolation. To understand if TGF $\beta 1$ gene expression was higher in genotype $\Pi$ EoE patient fibroblasts, we compared baseline TGF $\beta 1$ mRA, as well as mRA levels of TGF $\beta 1$ target genes. Genotype $\Pi$ fibroblasts had significantly higher baseline TGF $\beta 1$ gene expression as compared with CC genotype fibroblasts $(p<0.05$, Fig. ia). Genotype $\Pi \pi$ fibroblasts also had significantly higher baseline expression of the TGF $\beta 1$-target genes collagen $1 \mathrm{a} 1$ and MMP2 as compared with genotype CC fibroblasts $\left(p<0.05\right.$, Fig. Ib, c) ${ }^{30}$ A number of additional TGF $\beta 1$-target genes trended toward significantly higher baseline expression in ginotype TT fibroblasts including periostin, ${ }^{31}$ SERPINE1/Plasminogen Activator Inhibitor-1 (PAl-1), ${ }^{32}$ and fibronectin ( $p=0.06$ for each). In addition, collagen $1 a 1$ protein was elevated in $\Pi$ fibroblasts upon immunoblotting of whole-cell extracts (not shown). These data suggest inherent differences in fibrotic gene expression in esophageal fibroblasts from patients with CC versus $\Pi T$ genotype.
We hypothesized that due to the chronic elevated local exposure to TGF $\beta 1$ in genotype $\Pi$ patients and the elevated baseline gene expression of a subset of pro-fibrotic genes, esophageal fibroblasts from $\Pi$ subjects would not be capable of increasing pro-fibrotic gene expression following TGF $\beta 1$ treatment to the same extent as esophageal fibroblasts derived from CC genotype subjects. Indeed, TGF $\beta 1$ induced significantly higher levels of gene expression of collagen 1a1, periostin, PAI-1, and TGF $\beta 1$ itself, in genotype CC as compared with TT fibroblasts $(p<0.05$ for all) (Fig. $1 \mathrm{~d}-\mathrm{g}$ ), suggesting that lower in vive TGF $\beta 1$ exposure allowed for increased TGF $\beta 1$ responsivity in vito. To further demonstrate this point, we treated genotype CC esophagal fibroblasts from EoE subjects with TGF $\beta 1$ for up to $120 \mathrm{~h}$. CC fibroblasts had collagen $1 \mathrm{a} 1$ induction initially, but after $72 \mathrm{~h}$, they demonstrated a decrease in their response (Fig. Ii). These data are consistent with the hypothesis that tonic in vive TGF $\beta 1$ exposure is a potential mechanism for decreased $\Pi$ responsivity to exogenous TGF $\beta 1$.

In order to assess functional differences in the baseline activity of $\Pi T$ versus CC fibroblasts, we performed contraction and woundhealing assays. $\Pi$ fibroblasts had decreased contractility as compared with genotype CC fibroblasts (Fig. 1j). TT fibroblasts also trended toward increased wound healing as compared with

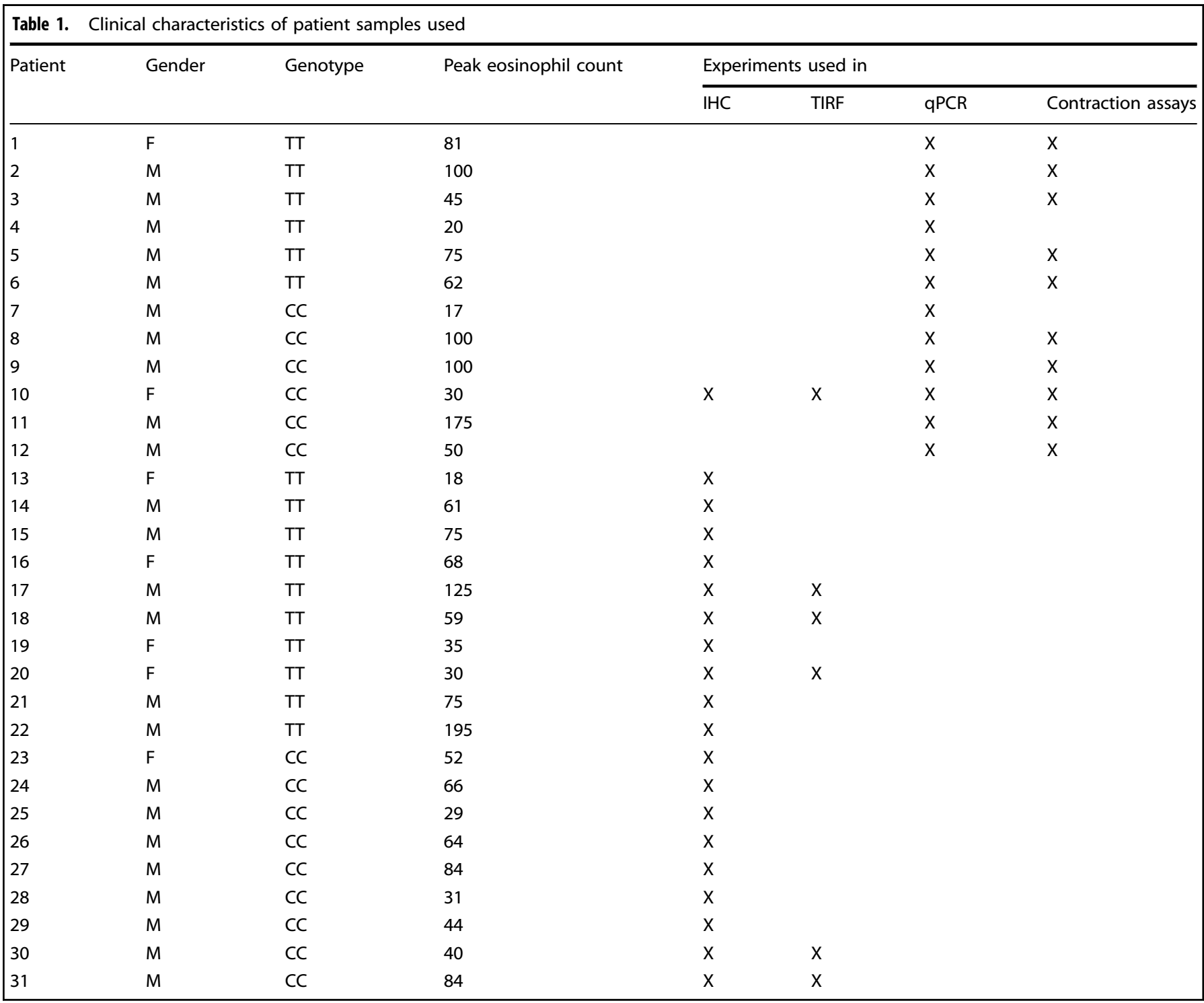

Mucosal Immunology (2020) 13:110-117

SPRINGER NATURE

SOCIETY FOR MUCOSA IMMUNOLOGY 

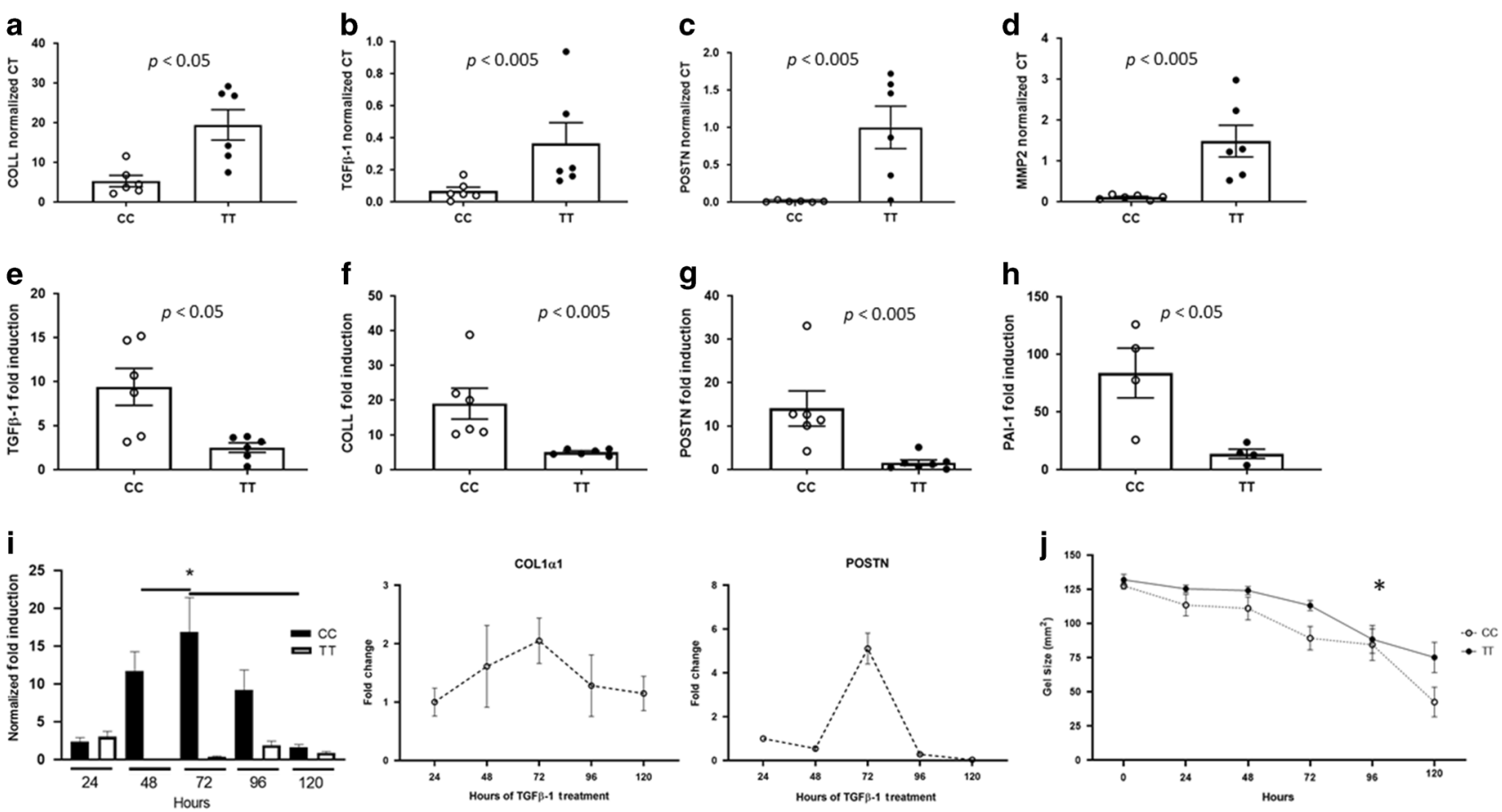

Fig. 1 Genotype CC and TT fibroblasts have different transcription profiles. Baseline transcript levels for TGF $\beta 1$ a, collagen $1 \alpha 1$ (COLL) b, periostin c, and matrix metalloproteinase 2 (MMP2) d in CC and TT primary esophageal fibroblasts matched for passage number, culture conditions, and EoE disease activity. TGF $\beta 1$-induced gene expression of TGF $\beta 1$ e, collagen $1 \alpha 1$ (COLL) f, periostin (POSTN) g, and plasminogen activator inhibitor-1 (PAI-1) h. All gene expressions were normalized to the housekeeping gene ribosomal protein L13a (RPL13a) and shown as normalized $\mathrm{Ct} \mathbf{a}-\mathbf{d}$ or as fold induction $\mathbf{e}-\mathbf{h}$ for the matched vehicle-treated well. Each data point represents a distinct patient. Each experiment was repeated in biological triplicates on different days and in qPCR technical replicates (2-3). Chronic exposure to TGF $\beta 1$ in CC versus TT (representative assay, left panel i) and over time in CC fibroblasts (right panel, $\mathbf{i} ; n=5$ different individuals, bars represent SEM). ${ }^{*} p<$ 0.01 . Contraction of TT versus CC fibroblasts done in triplicate wells in esophageal fibroblasts from ten different individuals $(n=5$ CC and $n=$ 5 TT) j. ${ }^{*} p<0.05$

genotype CC (Supplementary Fig. 1). These data demonstrate that the genotype at the TGF $\beta 1$ promoter SNP -509 associates with altered fibroblast function beyond transcriptional regulation.

\section{C-509T genotype and TGF $\beta 1$ affect esophageal epithelial barrier} function

We have reported that $\Pi$-genotype subjects exhibit more severe epithelial remodeling on routine histology as assessed by scoring epithelial features of basal zone hyperplasia + dilated intercellular spaces + desquamation as compared with genotype CC patients. ${ }^{20}$ For this reason, we hypothesized that esophageal epithelial barrier protein expression would be more aberrant in TT as compared with CC patients. We stained esophageal biopsies procured during active EoE from subjects of CC and TT genotypes for E-cadherin or claudin1. Quantification of membrane-bound E-cadherin demonstrated that TT-genotype subjects had less overall E-cadherin expression and significantly lower levels of properly localized E-cadherin as compared with CC genotype subjects $(p<0.01)$ (Fig. 2a, b). Consistent with the fact that proper claudin-1 localization depends on E-cadherin, there was also significantly less membrane-bound claudin-1 in genotype $\Pi$ as compared with genotype CC subjects $(p<0.01)$ (Fig. 2c, d). The degree of membrane-bound E-cadherin and claudin-1 expression positively correlated with one another regardless of genotype $(r=0.71, p<0.001)$, consistent with their interdependent localization patterns (Fig. 2e).

We used six active EoE biopsies ( $n=3 \mathrm{CC}, n=3 \mathrm{TT}$ ) in highresolution TIRF microscopy to understand the distribution of $\mathrm{E}$ cadherin clusters at $180-\mathrm{nm}$ resolution. The TIRF imaging technique allows observation of single-molecule fluorescence and determination of cluster sizes at the surfaces and boundaries of cells, while minimizing background fluorescence from the rest of the tissue sample. TIRF provides near-field (evanescent wave field) excitation, and only a few hundred nanometers of the specimen at the surface of the coverslip contributes to the fluorescent image. The resulting data demonstrated greater cluster size variability in $\Pi$-genotype subjects as compared with CC subjects. While CC subjects had a tighter distribution of Ecadherin clusters averaging $372 \pm 52 \mathrm{~nm}$, TT patient biopsies had a wider distribution of E-cadherin cluster sizes that averaged $446 \pm$ $189 \mathrm{~nm}$ (Fig. 3a, c). In addition, the size of the central core within an E-cadherin cluster was larger and had a wider size distribution in $\Pi$ as compared with CC patients (Fig. 3b). Together, these data demonstrate substantial differences in E-cadherin cluster structure at the nanometer level dependent on patient genotype.

If the $\Pi$ genotype causes increased local TGF $\beta 1$ exposure, which in turn alters epithelial barrier protein expression, then treatment of primary esophageal epithelial cells with TGF $\beta 1$ should mimic the in vivo state and disrupt E-cadherin localization and epithelial barrier function. We isolated primary esophageal epithelial cells from normal esophagi of organ donors. We cultured these non-transformed human epithelial cells on transwells, stratified them at air-liquid interface, until they generated an adequate barrier to electrical current, and then treated the cells with TGF $\beta 1$. Treatment with TGF $\beta 1$ for 2-7 days caused a significant decrease in transepithelial resistance in TGF $\beta 1$-treated versus vehicle-treated wells $(p<0.001)$. E-cadherin localization pattern was also disrupted in the stratified epithelial cells, such that untreated control wells had membrane-localized E-cadherin, while TGF $\beta 1$-treated wells had a disrupted pattern of E-cadherin localization (Fig. 4a, b).

Culture of primary esophageal epithelial cells in Matrigel induces the formation of three-dimensional esophageal epithelial cell spheroids, reminiscent of the complex structure of stratified epithelium. ${ }^{33,34}$ To understand the effects of TGF $\beta 1$ on E-cadherin 

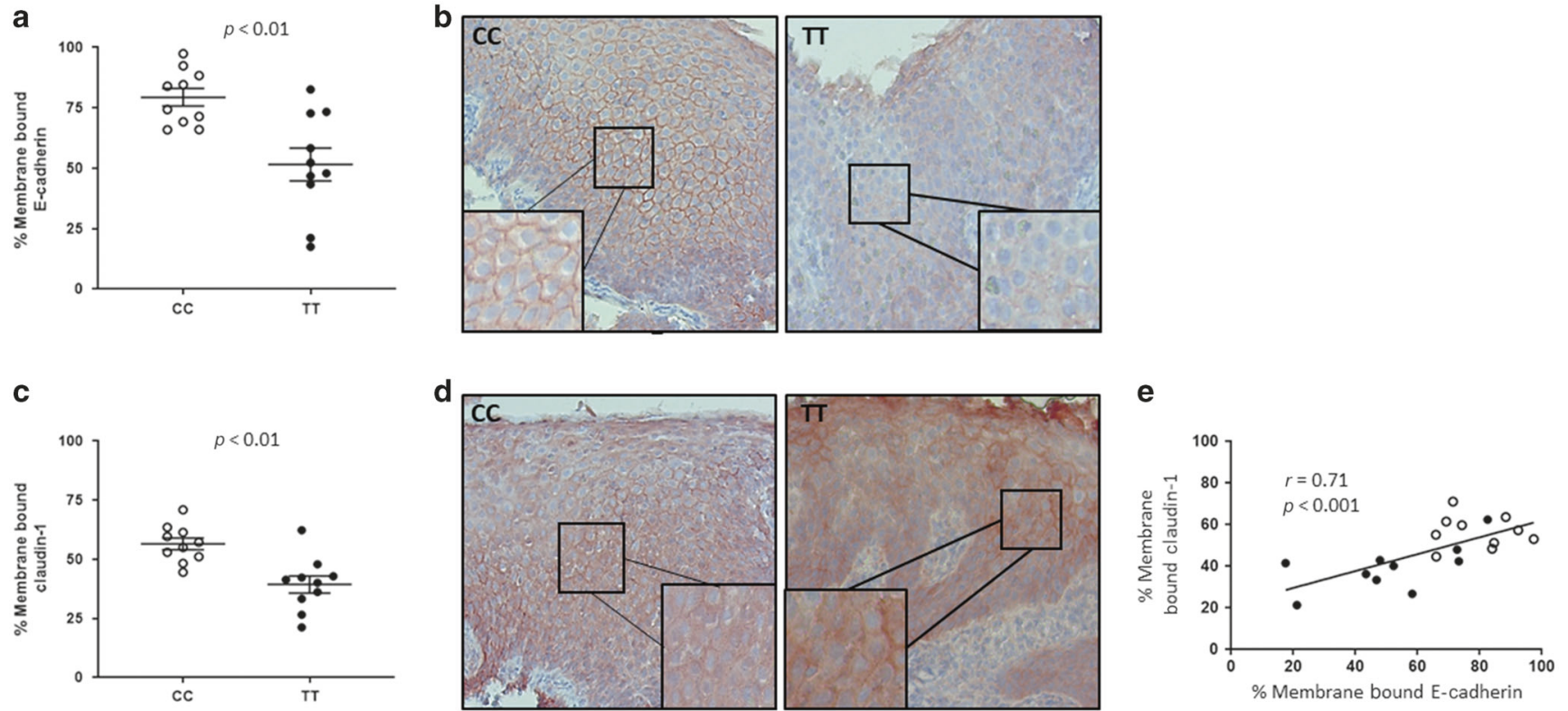

Fig. 2 Genotype CC and TT esophageal biopsies have distinct patterns of cell adhesion proteins. The percentage of cells per total epithelial height that expressed membrane-bound E-cadherin-1 a or claudin-1 c was quantified by using immunohistochemistry (red stain) and Image J analysis. Representative images of CC and TT subject epithelium are shown $\mathbf{b}$, $\mathbf{d}$. Each point on the graphs represents the average of five different epithelial regions in ten different subject biopsies with stains repeated on three different days. The degree of membrane-bound $\mathrm{E}$ cadherin and claudin-1 correlates positively regardless of genotype (closed circles $=\mathrm{TT}$, open circles $=\mathrm{CC}$ ) e
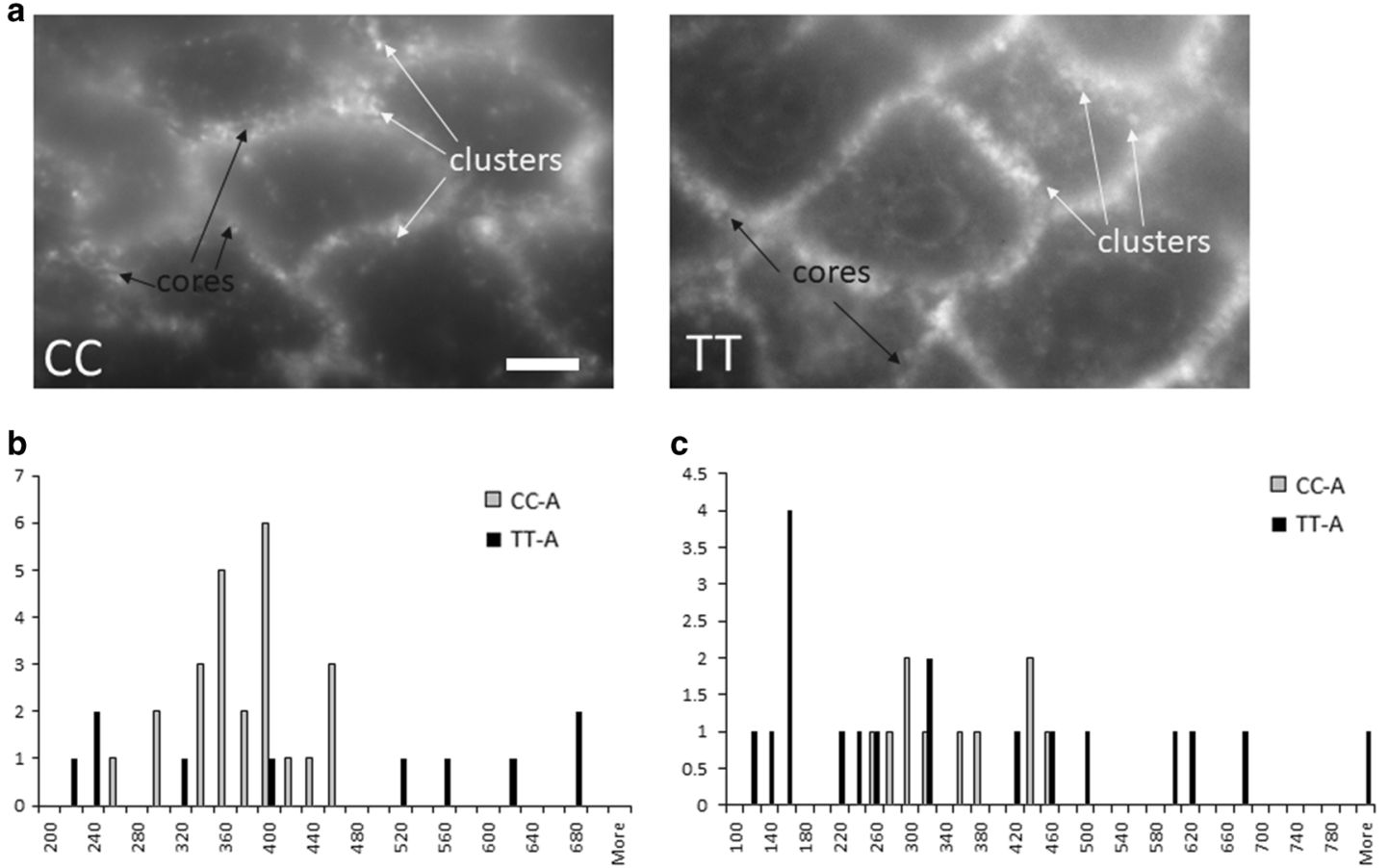

Fig. 3 Total internal reflection fluorescence (TIRF) imaging shows differences in CC and TT E-cadherin. TIRF high-resolution imaging of CC and TT-genotype biopsies a demonstrates more variability in the central core $\mathbf{b}$ and cluster $\mathbf{c}$ sizes in active genotype TT biopsies. Graphs represent quantification by using TIRF imaging of core and cluster sizes and distances in at least 20 images (5-6 areas per tissue section that had the sharpest focus) from six different subjects. Representative image is shown. Scale bar $=5$ microns

expression in epithelial cells in a three-dimensional architecture, we treated spheroids with TGF $\beta 1$ or vehicle. TGF $\beta 1$ caused mislocalization and decreased membrane expression of $\mathrm{E}$ cadherin in esophageal epithelial spheroids $(p<0.0001$ as compared with vehicle) (Fig. $4 c, d$ ). Concurrent treatment with the TGF $\beta 1$ receptor I/AlkV inhibitor SB431532 largely reversed the effect of TGF $\beta 1$ on E-cadherin localization (Fig. 4c, d), suggesting that the effects of TGF $\beta 1$ were occurring via a canonical signaling pathway. Together, our data suggest a genotype-influenced pathway, whereby $\Pi$-genotype fibroblasts and inflammatory cells $^{29,35}$ elevated local TGF $\beta 1$ exposure, and promoted Ecadherin disruption and dysregulated barrier function (Fig. 5). 

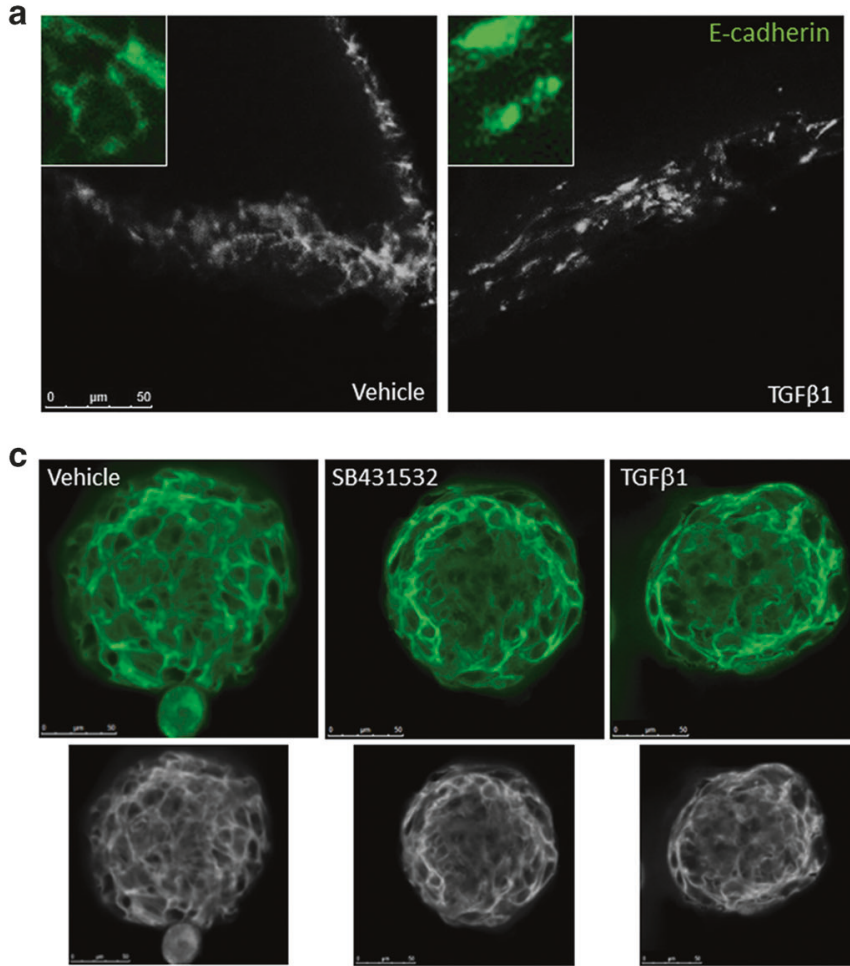
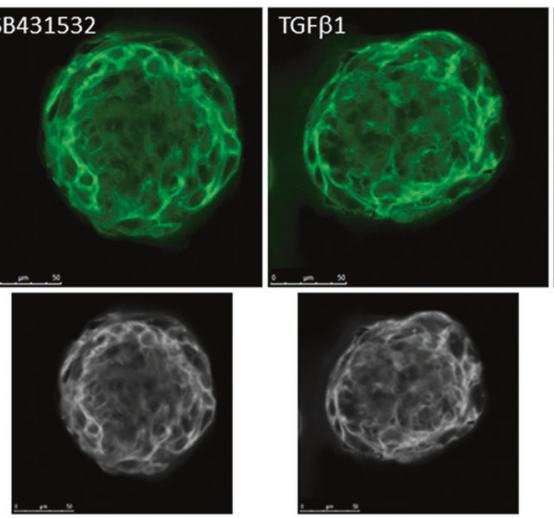

b
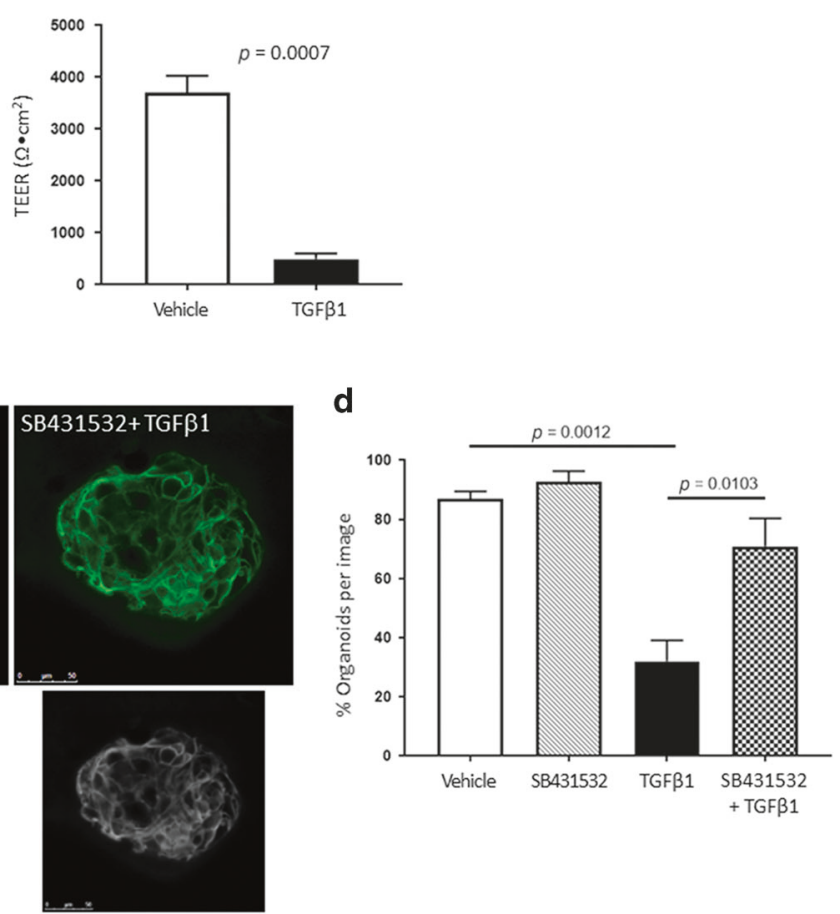

Fig. 4 TGF $\beta 1$ alters epithelial resistance and E-cadherin. Representative images of vehicle- and TGF $\beta 1$-treated epithelial cells on transwells $\mathbf{a}$ and the effect on transepithelial electrical resistance (TEER) b. Quantification of the percent of spheroids with properly localized E-cadherin during treatment with vehicle, the ALKV inhibitor SB431532, TGF $\beta 1$, or TGF $\beta 1+$ SB431532 c. Representative image of spheroids treated with vehicle, SB431532, TGF $\beta 1$, or both. Images are converted to grayscale below. Each image is a single slice of a three-dimensional confocal image $\mathbf{d}$. Spheroids were treated in 2-3 replicate wells by using two different donors on two different days. TEER experiments were repeated three times on different days in triplicate wells by using epithelial cells from two different donors. Bars represent means and standard error from a representative experiment

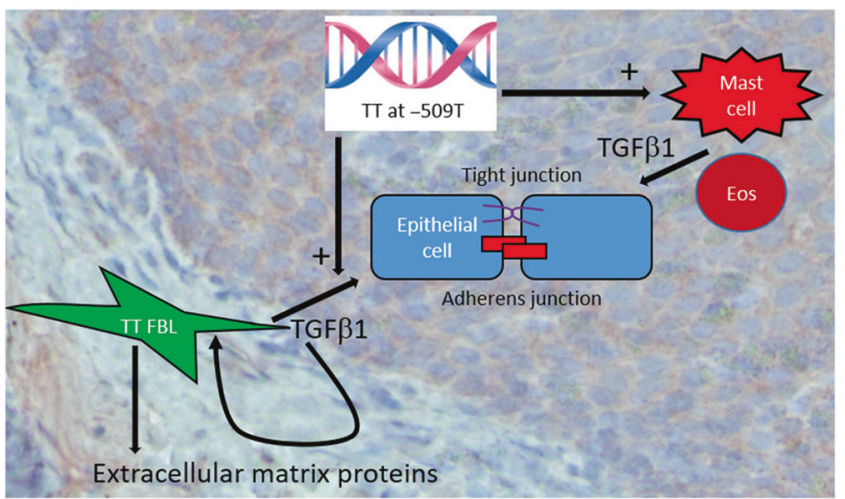

Fig. 5 Model of the effects of TT genotype at TGF $\beta 1$ SNP-509 on EoE remodeling. Genotype TT at -509 in the TGF $\beta 1$ promoter causes increased TGF $\beta 1$ expression from resident esophageal fibroblasts and associates with increased numbers of mast cells, which, along with eosinophils, are reservoirs of TGF $\beta 1$. TGF $\beta 1$ functions in an auto- or paracrine manner on resident fibroblasts to augment production of pro-fibrotic factors, such as collagen $1 \alpha 1$, especially in genotype TT EoE patients. TGF $\beta 1$ perpetuates EoE by causing barrier breakdown of both adherens and tight junctions

\section{DISCUSSION}

There is mounting evidence that EoE is a disease in which pathogenesis largely depends on intact barrier function for disease instigation and propagation. ${ }^{17,36,37}$ The $\Pi$ genotype at -509 in the TGF $\beta 1$ results in increased local TGF $\beta 1$ expression with increased numbers of TGF $\beta 1$-positive cells in the active EoE esophagus. ${ }^{24,38,39}$ Herein we demonstrate that fibroblasts from EoE children of the $\Pi$ genotype transcribe more TGF $\beta 1$, that TGF $\beta 1$ alters E-cadherin localization and epithelial barrier function, and that children with the $\Pi$ genotype have altered E-cadherin expression at nanometer resolution in the esophagus.

TGF $\beta 1$ has pleotropic effects on esophageal structural cells. It can disrupt esophageal epithelial cell barrier function, claudin-7 expression, and induce epithelial mesenchymal transition. ${ }^{25,40}$ In addition, TGF $\beta 1$ induces esophageal epithelial, fibroblast, and myocyte contraction along with myofibroblast transformation and smooth muscle cell hypertrophy. ${ }^{27,28,41}$ Herein, we used esophageal fibroblasts derived from genotype $\Pi$ children to understand intrinsic differences in fibroblast gene expression from CC genotype fibroblasts at baseline and in response to TGF $\beta 1$. TT-genotype fibroblasts had elevated baseline transcription of TGF $\beta 1$ and its target genes collagen $1 a 1$ and MMP2. Consistent with the increased TGF $\beta 1$ exposure in vivo in $T$ subjects, CC fibroblasts had a more robust induction of TGF $\beta 1$ target gene expression following exposure to the cytokine in vitro. Genotype $\Pi$ esophageal fibroblasts also demonstrated altered contractile properties, suggesting that they may be more fibrotic but less contractile than genotype CC esophageal fibroblasts. Further, we propose that in vivo, $\Pi$ fibroblasts producing more TGF $\beta 1$ would cause a more profound mislocalization of barrier proteins, resulting in a more severe disease phenotype in terms of treatment and histology (Fig. 5). As such, EoE states that associate with increased TGF $\beta 1$ may predispose to a more severe disease phenotype. We have previously shown this to be the case in terms of tissue histology in food-sensitized, $\Pi$ genotype subjects. In addition, it has been demonstrated that EoE patients have mislocalized E-cadherin even while on topical 
corticosteroids. ${ }^{42}$ The extent of this adherens junction disruption may be dependent on local TGF $\beta 1$ exposure that can vary among patients. Our study does have the limitation that our experiments were not conducted by using epithelial cells from genotype $\Pi$ versus CC subjects. However, the numbers of epithelial cells isolated per biopsy specimen and the relative rarity of genotype $\Pi(10-12 \%)$ in the population limits our ability to readily complete such studies.

Not all EoE patients follow the same disease course nor do they have equal response to therapy. ${ }^{9,11,12,43,44}$ Although most patients have recurrent disease, the time to disease recrudescence can vary substantially among patients, and the initial response to therapy is variable. In addition, topical corticosteroids, despite reducing eosinophilic inflammation, may not completely resolve E-cadherin localization or barrier function. ${ }^{42}$ An incompletely healed or more severely damaged epithelial barrier may predispose to harder-totreat disease or EoE that is prone to recurrence. Recent data demonstrated that SPINK7 dysregulation unleashes a spontaneous alteration in esophageal barrier function with poor E-cadherin localization and expression. ${ }^{17}$ These data support a critical role of E-cadherin function in the esophageal mucosa and in EoE. Further insults to E-cadherin localization would only worsen this process in patients with dysregulated TGF $\beta 1$ expression, such as those with Loeys-Dietz, Marfan's, or TT genotype at C-509T. It is interesting to suggest that a sub-phenotype of EoE patients may benefit from the concurrent use of a localized TGF $\beta 1$-inhibiting therapy. Given the ability to treat the esophagus topically, such an approach may diminish unwanted side effects of global TGF $\beta 1$ inhibition. It would be of great utility to understand if therapies that are primarily aimed at protecting epithelial barrier breakdown and normalizing E-cadherin localization are efficacious in EoE. Further, it would be of interest to understand if TGF $\beta 1$ blockade during a progressed disease state could help decrease fibrotic gene expression and its clinical sequelae. Our data suggest that such a therapeutic approach may be worth considering for clinical trials.

\section{METHODS}

EoE subjects/biopsies and immunohistochemistry

By using our University of California, San Diego (UCSD)/Rady Children's Hospital, San Diego (RCHSD) EoE database, we selected a cohort of EoE subjects who had active EoE, defined as $\geq 15$ eosinophils per hpf. Written informed consent/assent was obtained under UCSD/RCHSD IRB-approved protocol 091485. Normal esophageal epithelial cells were isolated from cadaveric/ organ donor esophagi, and were not considered human subjects research but approved under UCSD IRB protocol 130835.

Hematoxylin- and eosin-stained, formalin-fixed, paraffinembedded specimens were scored by a single pathologist (R.N.) by using our previously published standardized histology scoring tool. ${ }^{45}$ The numbers of epithelial eosinophils, the severity of basal zone hyperplasia (scored 0-3), the presence/absence of dilated intercellular spaces (scored 0/1), and the presence/absence of epithelial desquamation (scored 0/1) were quantified. The epithelial remodeling score is a composite index of the latter three epithelial cell features (maximum score of 5).

Paraffin-embedded biopsy specimens were processed for immunohistochemistry as previously described. ${ }^{46}$ E-cadherin (Abcam, Cambridge, MA, 1:100) and claudin-1 (Abcam, Cambridge, MA, 1:300) staining was quantified by using color detection and image analysis (ImageJ) to assess cell layers with membrane bound versus cytoplasmic E-cadherin and claudin-1 relative to the total epithelial height. For any stain, 3-5 image fields were captured, and the mean value for a given subject was utilized. All images were analyzed under identical light microscopic conditions, including magnification, gain, camera position, and background illumination.
Total internal reflection fluorescence (TIRF) microscopy For high-contrast images of fluorescently stained tissue, we used an inverted Olympus IX83 microscope with a total internal reflection fluorescence objective (UAPON 100X/1.49NA, Olympus), sCMOS camera (ORCA_Flash4.0 V3, Hamamatsu), and 488-nm excitation laser (Genesis MX488, Coherent). In order to ensure that $\mathrm{E}$-cadherin distribution was being quantified at the same region of the epithelium, the tissue was evaluated initially under $\times 10$ magnification to identify mid-epithelial areas of flat tissue located close to the coverslip. We then chose sharply in focus parts of tissue areas and measured the full width at half maximum (FWHM) of E-cadherin clusters, and the size of the nonfluorescent, dark core within an E-cadherin cluster.

Quantitative PCR and genotyping

Fibroblasts were collected in RNA Stat-60 (Tel Test Inc., Friendswood, TX) for RT qPCR experiments as previously described. ${ }^{28}$ Primer sequences were TGF $\beta 1$ forward GGTGGAAACCCA CAACGA AATC, reverse AATTCCCCTCCACGGCTCAAC; collagen I forward CAGCCGCTTCACCTACAGC, reverse TITGTATTCAATCACTGTCTTG CC; matrix metalloproteinase 2 (MMP2) forward AGCTCTATGGGG CCTCTCCTGA, reverse TCACCAC GGATCTGAGCGATGC; plasminogen activator inhibitor-1 (PAI-1) forward TGGTTCTGCC CAAGTTC TCCCTG, reverse TGCCACTCTCGTTCACCTCG; periostin forward GTCTITGAGA CGCTGGAAGG, reverse AGATCCGTGAAGGTGGTTTG; fibronectin forward GGAGAATT CAAGTGTGACCCTCA, reverse TGC CACTGTTCTCCTACGTGG; RPL13A forward CATA GGAAGCTGGG AGCAAG, reverse GCCCTCCAATCAGTCTTCTG.

Genomic DNA was extracted from whole blood, peripheral blood mononuclear cells, or esophageal fibroblasts by using QIAmp genomic DNA extraction kit; Genotype at -509 at the TGF $\beta 1$ promoter was determined according to the manufacturer's instructions for PCR-based SNP analysis (Thermo Fisher SNP ID: rs1800469).

Cell culture, epithelial cell spheroids, and treatment

Human organ transplant donor esophagi provided by the National Disease Research Interchange and the Arkansas Regional Organ Recovery Agency were processed to isolate epithelial cells. EoE biopsies were processed to isolate fibroblasts. ${ }^{28}$ Cell identity was confirmed by proper morphology and cell-relevant gene expression. All fibroblast lines were cultured in SMCM complete media (ScienCell, San Diego, CA) and matched for passage and disease activity state of the patients (Table 1). Fibroblasts were used at passage $7 \pm 1$ passage. Cells were treated with TGF $\beta 1(10 \mathrm{ng} / \mathrm{mL})$ for $48 \mathrm{~h}$ for $\mathrm{qPCR}$ experiments and for up to $120 \mathrm{~h}$ with media and TGF $\beta 1$ replacement every other day for chronic exposure experiments.

Epithelial cells were isolated from human donor cadaveric esophagi, suspended in Matrigel (Corning \#356231), and cultured for 5-7 days in a defined medium (advanced DMEM/F12, Thermo Fisher \#12634010), HEPES (20 mM, Thermo Fisher \# 15630080), glutamine (Thermo Fisher \#A1286001), N-2, B-27, N-Acetyl-Lcysteine $(500 \mathrm{ng} / \mathrm{mL}$, Sigma-Aldrich \#A7250), r-spondin $(5 \mu \mathrm{g} /$ $\mathrm{mL})$, noggin $(100 \mathrm{ng} / \mathrm{mL}$, PeproTech \#250-38), EGF $(500 \mu \mathrm{g} / \mathrm{mL}$, Thermo Fisher \#PHG0311), and rho kinase inhibitor (Y27632, 1.2 $\mu \mathrm{g} / \mathrm{mL}$, LC Laboratories \#Y-5301) replaced every other day and assessed for growth of epithelial spheroids prior to treatment with TGFß1 (10 ng/mL) and/or ALKV Inhibitor SB431532 (Invivogen \#inh-sb43) for $48 \mathrm{~h}^{33}$ After treatment, spheroids were immunofluorescently stained for E-Cadherin (Abcam \#ab1416), and the fluorescent signal was amplified by tyramide signal amplification (Thermo Fisher \#T20912). All images were captured by using identical brightness, gain, and fluorescence microscope settings.

Contraction assays

$C C$ and $\Pi$ patient fibroblasts were cultured in gels consisting of DMEM (Thermo Fisher Scientific \#12100061), sodium bicarbonate 
(Thermo Fisher Scientific \#25080094), and collagen (Advanced BioMatrix \#5005-100ML), and treated with recombinant TGF $\beta 1$ $(10 \mathrm{ng} / \mathrm{mL}$ ) over 5 days. Images were captured daily on a hemiDoc transilluminator (BioRad Laboratories), and gel area was quantified by using ImageJ.

\section{Transepithelial resistance}

Epithelial cells were plated on transwell-permeable supports (12$\mathrm{mm}$ insert, 0.4- $\mu \mathrm{m}$ polyester membrane, Costar, Cambridge, MA) coated with collagen $(0.033 \mathrm{mg} / \mathrm{mL})$, human fibronectin $(0.01 \mathrm{mg} /$ $\mathrm{mL})$, and BSA $(0.02 \mathrm{mg} / \mathrm{mL}){ }^{47}$ Resistance was measured daily by using a voltohmmeter (World Precision Instruments, Sarasota, FL), and measurements were normalized to the insert area by subtracting the resistance of a blank insert with no cells. Epithelial resistance studies were repeated three times on different days each in triplicate wells by using esophageal epithelial cells derived from two different donors. Cells were treated with recombinant TGF $\beta 1$ for 2-7 days.

Statistical analysis

Statistical analyses were performed by using GraphPad Prism (San Diego, CA). Statistical analysis of data was performed by Student's $t$ test or Mann-Whitney test where appropriate. A Spearman's coefficient was calculated for correlations. A two-tailed $p$-value $<0.05$ was considered statistically significant.

\section{ACKNOWLEDGEMENTS}

We thank the Aceves laboratory members for technical assistance, and the patients and their families for participating in the study. We also thank NIH/NIAID AI092135 (S.S.A., R.K.), NIH/NIAID Al135034 (S.S.A.), and NIH/NCRR/NCATS UL1TR000039 (R.K.).

\section{AUTHOR CONTRIBUTIONS}

L.D.D.: Designed, performed, and analyzed experiments and data, assisted in paper preparation, and critically reviewed the paper. R.R.: Designed, performed, and analyzed experiments and data, assisted in paper preparation, and critically reviewed the paper. A.B.: Designed, performed, and analyzed experiments and data, assisted in paper preparation, and critically reviewed the paper. M.C.M.: Designed, performed, and analyzed experiments and data, assisted in paper preparation, and critically reviewed the data. R.O.N.: Analyzed data, provided essential expertise, and critically reviewed the paper. R.D.: Provided samples, assisted with experimental design, analyzed data, and critically reviewed the paper. Z.L.: Provided essential expertise, analyzed data, and critically reviewed the paper. K.B.: Provided essential expertise, analyzed data, and critically reviewed the paper. R.K.: Provided samples, analyzed data, and critically reviewed the paper. S.S.A.: Designed experiments, procured funding, analyzed experimental data, and wrote the paper.

\section{ADDITIONAL INFORMATION}

The online version of this article (https://doi.org/10.1038/s41385-019-0214-9) contains supplementary material, which is available to authorized users.

Competing interests: The authors declare no competing interests.

Publisher's note Springer Nature remains neutral with regard to jurisdictional claims in published maps and institutional affiliations.

\section{REFERENCES}

1. Dellon, E. S. et al. Updated international consensus diagnostic criteria for eosinophilic esophagitis: Proceedings of the AGREE Conference. Gastroenterology. 155, 1022-1033 (2018).

2. Liacouras, C. A. et al. Eosinophilic esophagitis: updated consensus recommendations for children and adults. J. Allergy Clin. Immunol. 128, 3-20e6 (2011). quiz 1-2.

3. Dellon, E. S. Epidemiology of eosinophilic esophagitis. Gastroenterol. Clin. North Am. 43, 201-218 (2014).

4. Spergel, J. M. et al. Summary of the updated international consensus diagnostic criteria for eosinophilic esophagitis: AGREE conference. Ann. Allergy Asthma Immunol. 121, 281-284 (2018).
5. Schoepfer, A. M. et al. Delay in diagnosis of eosinophilic esophagitis increases risk for stricture formation in a time-dependent manner. Gastroenterology 145, 1230-6 e1-2 (2013).

6. Kwiatek, M. A. et al. Mechanical properties of the esophagus in eosinophilic esophagitis. Gastroenterology 140, 82-90 (2011).

7. Nicodeme, F. et al. Esophageal distensibility as a measure of disease severity in patients with eosinophilic esophagitis. Clin. Gastroenterol. Hepatol. 11, 1101-7 e1 (2013).

8. Dellon, E. S. et al. A phenotypic analysis shows that eosinophilic esophagitis is a progressive fibrostenotic disease. Gastrointest. Endosc. 79, 577-85 e4 (2014).

9. Greuter, T. et al. Long-term treatment of eosinophilic esophagitis with swallowed topical corticosteroids: development and evaluation of a therapeutic concept. Am. J. Gastroenterol. 112, 1527-1535 (2017).

10. Greuter, T. et al. Maintenance treatment of eosinophilic esophagitis with swallowed topical steroids alters disease course over a 5-year follow-up period in adult patients. Clin. Gastroenterol. Hepatol. 112, 1527-1535 (2018).

11. Rajan, J. et al. Long-term assessment of esophageal remodeling in patients with pediatric eosinophilic esophagitis treated with topical corticosteroids. J. Allergy Clin. Immunol. 137, 147-56 e8 (2016).

12. Eluri, S. et al. The extremely narrow-caliber esophagus is a treatment-resistant subphenotype of eosinophilic esophagitis. Gastrointest. Endosc. 83, 1142-1148 (2016).

13. Fahey, L. M. et al. Food allergen triggers are increased in children with the TSLP risk allele and eosinophilic esophagitis. Clin. Transl. Gastroenterol. 9, 139 (2018).

14. Rothenberg, M. E. et al. Common variants at $5 q 22$ associate with pediatric eosinophilic esophagitis. Nat. Genet. 42, 289-291 (2010).

15. Kottyan, L. C. et al. Genome-wide association analysis of eosinophilic esophagitis provides insight into the tissue specificity of this allergic disease. Nat. Genet. 46, 895-900 (2014).

16. Davis, B. P. et al. Eosinophilic esophagitis-linked calpain 14 is an IL-13-induced protease that mediates esophageal epithelial barrier impairment. $J \mathrm{Cl}$ Insight 1, e86355 (2016).

17. Azouz, N. P. et al. The antiprotease SPINK7 serves as an inhibitory checkpoint for esophageal epithelial inflammatory responses. Sci. Transl. Med. 10, 444 (2018).

18. Frischmeyer-Guerrerio, P. A. et al. TGFbeta receptor mutations impose a strong predisposition for human allergic disease. Sci. Transl. Med. 5, $195 \mathrm{ra} 94$ (2013).

19. Abonia, J. P. et al. High prevalence of eosinophilic esophagitis in patients with inherited connective tissue disorders. J. Allergy Clin. Immunol. 132, 378-386 (2013).

20. Rawson, R. et al. The TGFbeta1 promoter SNP C-509T and food sensitization promote esophageal remodeling in pediatric eosinophilic esophagitis. PLoS One 10, e0144651 (2015).

21. Aceves, S. S. et al. Resolution of remodeling in eosinophilic esophagitis correlates with epithelial response to topical corticosteroids. Allergy 65, 109-116 (2010).

22. Sharma, S. et al. Variants in TGFB1, dust mite exposure, and disease severity in children with asthma. Am. J. Respir. Crit. Care Med. 179, 356-362 (2009).

23. Salam, M. T., Gauderman, W. J., McConnell, R., Lin, P. C. \& Gilliland, F. D. Transforming growth factor- 1 C-509T polymorphism, oxidant stress, and early-onset childhood asthma. Am. J. Respir. Crit. Care Med. 176, 1192-1199 (2007).

24. Silverman, E. S. et al. Transforming growth factor-beta1 promoter polymorphism C-509T is associated with asthma. Am. J. Respir. Crit. Care Med. 169, 214-219 (2004).

25. Kagalwalla, A. F. et al. Eosinophilic esophagitis: epithelial mesenchymal transition contributes to esophageal remodeling and reverses with treatment. J. Allergy Clin. Immunol. 129, 1387-1396 e7 (2012).

26. Muir, A. B. et al. Eosinophilic esophagitis-associated chemical and mechanical microenvironment shapes esophageal fibroblast behavior. J. Pediatr. Gastroenterol. Nutr. 63, 200-209 (2016).

27. Muir, A. B., Wang, J. X. \& Nakagawa, H. Epithelial-stromal crosstalk and fibrosis in eosinophilic esophagitis. J. Gastroenterol. 54, 10-18 (2019).

28. Beppu, L. Y. et al. TGF-beta1-induced phospholamban expression alters esophageal smooth muscle cell contraction in patients with eosinophilic esophagitis. J. Allergy Clin. Immunol. 134, 1100-1107 e4 (2014).

29. Aceves, S. S. et al. Mast cells infiltrate the esophageal smooth muscle in patients with eosinophilic esophagitis, express TGF-beta1, and increase esophageal smooth muscle contraction. J. allergy Clin. Immunol. 126, 1198-204 e4 (2010).

30. Beppu, L. et al. MMPs-2 and -14 are elevated in eosinophilic esophagitis and reduced following topical corticosteroid therapy. J. Pediatr. Gastroenterol. Nutr. 61, 194-199 (2014).

31. Blanchard, C. et al. Periostin facilitates eosinophil tissue infiltration in allergic lung and esophageal responses. Mucosal Immunol. 1, 289-296 (2008).

32. Rawson, R. et al. TGF-beta1-induced PAI-1 contributes to a profibrotic network in patients with eosinophilic esophagitis. J. Allergy Clin. Immunol. 138, 791-800 e4 (2016). 
33. Whelan, K. A., Muir, A. B. \& Nakagawa, H. Esophageal 3D culture systems as modeling tools in esophageal epithelial pathobiology and personalized medicine. Cell Mol. Gastroenterol. Hepatol. 5, 461-478 (2018).

34. DeWard, A. D., Cramer, J. \& Lagasse, E. Cellular heterogeneity in the mouse esophagus implicates the presence of a nonquiescent epithelial stem cell population. Cell Rep. 9, 701-711 (2014).

35. Aceves, S. S., Newbury, R. O., Dohil, R., Bastian, J. F. \& Broide, D. H. Esophageal remodeling in pediatric eosinophilic esophagitis. J. Allergy Clin. Immunol. 119, 206-212 (2007)

36. Rochman, M., Azouz, N. P. \& Rothenberg, M. E. Epithelial origin of eosinophilic esophagitis. J. Allergy Clin. Immunol. 142, 10-23 (2018).

37. Rochman, M. et al. Profound loss of esophageal tissue differentiation in eosinophilic esophagitis. J. Allergy Clin. Immunol. 142, 10-23 (2017).

38. Che, Z. et al. The association between the C-509T and T869C polymorphisms of TGF-beta1 gene and the risk of asthma: a meta-analysis. Hum. Immunol. 75, 141-150 (2014)

39. Khalil, M. S., El Nahas, A. M. \& Blakemore, A. I. Transforming growth factor-beta1 SNPs: genetic and phenotypic correlations in progressive kidney insufficiency. Nephron Exp. Nephrol. 101, e31-e41 (2005).

40. Nguyen, N. et al. TGF-beta1 alters esophageal epithelial barrier function by attenuation of claudin-7 in eosinophilic esophagitis. Mucosal Immunol. 11, 415-426 (2018)
41. Muir, A. B. et al. Eosinophilic esophagitis associated chemical and mechanical microenvironment shapes esophageal fibroblast behavior. J. Pediatr. Gastroenterol. Nutr. (2015).

42. Simon, D. et al. Evidence of an abnormal epithelial barrier in active, untreated and corticosteroid-treated eosinophilic esophagitis. Allergy $\mathbf{7 3}$, 239-247 (2018).

43. Eluri, S. et al. Diminishing effectiveness of long-term maintenance topical steroid therapy in PPI non-responsive eosinophilic esophagitis. Clin. Transl. Gastroenterol. 8, e97 (2017)

44. Wolf, W. A. et al. Predictors of response to steroid therapy for eosinophilic esophagitis and treatment of steroid-refractory patients. Clin. Gastroenterol. Hepatol. 13, 452-458 (2015).

45. Aceves, S. S., Newbury, R. O., Dohil, M. A., Bastian, J. F. \& Dohil, R. A symptom scoring tool for identifying pediatric patients with eosinophilic esophagitis and correlating symptoms with inflammation. Ann. Allergy Asthma Immunol. 103, 401-406 (2009).

46. Doshi, A. et al. IL-9 alters epithelial barrier and E-cadherin in eosinophilic esophagitis. J. Pediatr. Gastroenterol. Nutr. 68, 225-231 (2019).

47. Chen, $X$. et al. Bile salts disrupt human esophageal squamous epithelial barrier function by modulating tight junction proteins. Am. J. Physiol. Gastrointest. Liver Physiol. 303, G199-G208 (2012). 